\title{
Diffusion-weighted whole-body imaging with background body signal suppression/T2-weighted image fusion of gastrointestinal cancers
}

\author{
MINORU TOMIZAWA ${ }^{1}$, FUMINOBU SHINOZAKI ${ }^{2}$, KAZUNORI FUGO $^{3}$, TAKAFUMI SUNAOSHI ${ }^{2}$, \\ DAISUKE KANO ${ }^{2}$, SATOMI TANAKA ${ }^{2}$, AIKA OZAKI ${ }^{2}$, ERIKO SUGIYAMA ${ }^{2}$, MISAKI SHITE ${ }^{2}$, \\ RYOUTA HAGA $^{2}$, AKIRA BABA ${ }^{2}$, YOSHIYA FUKAMIZU ${ }^{2}$, TOSHIYUKI FUJITA ${ }^{2}$, SATOSHI KAGAYAMA ${ }^{2}$, \\ RUMIKO HASEGAWA ${ }^{4}$, AKIRA TOGAWA ${ }^{4}$, YOSHINORI SHIRAI ${ }^{4}$, NOBORU ICHIKI ${ }^{4}$, YASUFUMI MOTOYOSHI ${ }^{5}$,

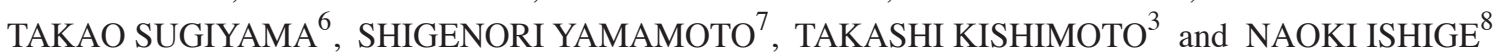

\begin{abstract}
Departments of ${ }^{1}$ Gastroenterology and ${ }^{2}$ Radiology, National Hospital Organization Shimoshizu Hospital, Yotsukaido, Chiba 284-0003; ${ }^{3}$ Department of Molecular Pathology, Chiba University Graduate School of Medicine, Chiba, Chiba 260-8670; Departments of ${ }^{4}$ Surgery, ${ }^{5}$ Neurology, ${ }^{6}$ Rheumatology, ${ }^{7}$ Pediatrics and ${ }^{8}$ Neurosurgery,

National Hospital Organization Shimoshizu Hospital, Yotsukaido, Chiba 284-0003, Japan
\end{abstract}

Received January 11,2016; Accepted April 27, 2016

DOI: $10.3892 / \mathrm{mco} .2016 .897$

\begin{abstract}
Diffusion-weighted whole-body imaging with background body signal suppression (DWIBS) yields positive results for cancer against the surrounding tissues. The combination of DWIBS and T2-weighted images (DWIBS/T2) in the diagnosis of gastrointestinal tract cancers was retrospectively analyzed in the present study. Patients were subjected to magnetic resonance imaging after cancer was diagnosed through specimens obtained via biopsy or endoscopic mucosal resection. Sixteen patients were assessed between July, 2012 and June, 2013 and the correlation between detection with DWIBS/T2 and $\mathrm{T}$ staging was analyzed. Regarding patients who underwent surgery, the correlation between detection with DWIBS/T2 and the diameter or depth of invasion was analyzed. All cancers that had advanced to $>\mathrm{T} 2$ stage were detectable by DWIBS/T2, whereas all cancers staged as $<\mathrm{T} 1$ were not $(\mathrm{P}<0.0001)$. Tumors that were undetected by DWIBS/T2 had a mean diameter of $1.53 \pm 0.25 \mathrm{~cm}$, whereas those detected had a mean diameter of $3.63 \pm 1.88 \mathrm{~cm}$; however, the difference was not statistically significant $(\mathrm{P}=0.1053)$. Cancers invading beyond the muscularis propria were detectable by DWIBS/T2, while those which had not invaded the mucosa were not $(\mathrm{P}=0.0476)$. In conclusion, DWIBS/T2 was able to positively identify gastrointestinal tract
\end{abstract}

Correspondence to: Dr Minoru Tomizawa, Department of Gastroenterology, National Hospital Organization Shimoshizu Hospital, 934-5 Shikawatashi, Yotsukaido, Chiba 284-0003, Japan E-mail: nihminor-cib@umin.ac.jp

Key words: diffusion-weighted whole-body imaging with background body signal suppression, T2-weighted image, diameter, depth of invasion cancers at an advanced stage ( $>\mathrm{T} 2)$ or invading beyond the muscularis propria.

\section{Introduction}

Endoscopy is the gold standard for diagnosing cancers of the gastrointestinal tract, including the esophagus, stomach, colon and rectum $(1,2)$. However, endoscopy is not suitable for evaluating the depth of invasion and extent of cancer, as it only allows observation of the lumen. Endoscopic ultrasonography (EUS) and contrast-enhanced computed tomography (CE-CT) are performed to assess the structure of the primary lesion, the depth of invasion into the surrounding tissues and distant metastasis $(3,4)$. Assessing the depth of invasion may occasionally be difficult due to the weak contrast of the cancer against the surrounding tissues. Therefore, an imaging modality with a strong signal and contrast would facilitate the assessment of the depth of tumor invasion.

Magnetic resonance imaging (MRI) is not as popular as CT due to blurring and low spatial resolution (5). However, MRI may be a promising method if a strong soft tissue contrast in the abdomen can be achieved. Diffusion-weighted wholebody imaging with background body signal suppression (DWIBS) images are acquired using multiple-signal averaging, pre-pulse fat suppression and heavy diffusion weighting during free breathing (6). DWIBS is based on diffusion-weighted imaging (DWI) that visualizes and assesses the random movement of water at the molecular level (Brownian motion) $(7,8)$. An advantage of DWIBS is that it provides a strong contrast of cancerous against surrounding non-cancerous tissues, which is useful for the detection, staging and monitoring of the response to therapy (9). A major limitation of DWIBS is that anatomical analysis may be difficult at times $(10,11)$. Fusion images of DWIBS and T2-weighted images (T2WI (DWIBS/T2) are created by overlapping DWIBS 
Table I. Pulse sequences used in the present study.

\begin{tabular}{lccc}
\hline Parameters & T1-weighted image & T2-weighted image & DWI (DWIBS/T2) \\
\hline Echo & GRE & Single-shot SE & EPI SE \\
TR (msec) & Shortest & 1,000 & 11,250 \\
TE $(\mathrm{msec})$ & First: 2.3 (out-phase), second, 4.6 (in-phase) & 90 & 83 \\
Flip angle $\left(^{\circ}\right)$ & 75 & 1 & 90 \\
NSA & 1 & 8 & 4 \\
Slice thickness (mm) & 8 & 1 & 5 \\
Slice gap & 1 & None & SPAIR \\
Fat saturation & None & Posterior-anterior & Posterior-anterior \\
Phase encoding direction & Posterior-anterior & 90 \\
\hline DWI, diffusion-weighted image; DWIBS/T2, diffusion-weighted whole-body imaging with background body signal suppression/T2-weighted \\
image fusion; GRE, gradient echo; SE, spin echo; EPI, echo planar imaging; SPAIR, spectral attenuated inversion recovery; TR, repetition \\
time; TE, echo time; NSA, number of signal averages.
\end{tabular}

with T2WI using a workstation $(9,12,13)$. DWIBS/T2 therefore clearly illustrates functional information in anatomical images.

In the present study, the performance of DWIBS/T2 in the diagnosis of gastrointestinal cancers was retrospectively analyzed.

\section{Patients and methods}

Ethical statement. The present study was approved by the Ethics Committee of the National Hospital Organization Shimoshizu Hospital (Yotsukaido, Japan). This was not considered a clinical trial, as the procedures were performed as a part of routine clinical practice. Written informed consent was obtained from all patients who were subjected to MRI, upper gastrointestinal endoscopy, colonoscopy and CE-CT. Consent was obtained from patients who were subjected to abdominal ultrasonography, but written form was waived. Written informed consent for inclusion into the study was also waived, as patient records were anonymized and retrospectively analyzed.

Study design. Patient records, including imaging, from July, 2012 until June, 2013 were retrospectively analyzed. The patients were subjected to upper gastrointestinal endoscopy to investigate abdominal pain, anemia, hematemesis and other symptoms suggesting diseases of the esophagus, stomach or duodenum. The patients were subjected to colonoscopy for the investigation of abdominal pain, melena and other symptoms suggesting diseases of the colon or rectum. A proportion of the patients had been subjected to upper gastrointestinal endoscopy and colonoscopy as part of screening. The patient inclusion criteria were as follows: i) Pathological diagnosis of esophageal, gastric or colon cancer based on bioptic or endoscopic mucosal resection specimens; ii) available DWIBS/T2 images. A total of 8 men (mean age, $71.6 \pm 12.5$ years; range 67-77) and 8 women (mean age, $71.6 \pm 4.0$ years; range, 46-82) were enrolled in the present study. The depth of invasion and tumor diameter were assessed based on specimens obtained through surgery or endoscopic mucosal resection. T staging was performed using CE-CT, abdominal ultrasonography or EUS, according to the 7th edition of the American Joint Committee on Cancer classification (14).

MRI. All MRI studies were performed using a 1.5 Tesla scanner (Achieva, software version 3.2.2, Philips Medical Systems, Best, The Netherlands). T1-weighted image (T1WI), T2WI and DWI were obtained with pulse sequences, as depicted in Table I. DWIBS/T2 images were constructed with Extended MR WorkSpace (Philips, Best, The Netherlands). The DWI gradients were applied along the $\mathrm{X}, \mathrm{Y}$ and $\mathrm{Z}$ axes before and after a $180^{\circ}$ inversion pre-pulse to obtain fat-saturated, isotropic images with DWI sensitivity using the following parameters for a single stack: b-value, $0 \mathrm{~mm}^{2} / \mathrm{sec}$ and $800 \mathrm{~mm}^{2} / \mathrm{sec}$; repetition time/echo time/inversion recovery, 6,960/79/150 msec; acquisition matrix, 176x115; and reconstruction matrix, 256; field of view: Right/left, $530 \mathrm{~mm}$; anterior/posterior, $349 \mathrm{~mm}$; and feet/head, $226 \mathrm{~mm}$; slice thickness, $6 \mathrm{~mm}$; size of reconstructed voxel, $2.07 \times 2.08 \times 6 \mathrm{~mm}^{3} ; 4$ averages. One radiologist and one gastroenterologist analyzed the DWIBS/T2 images. To rule out T2 shine-through or differentiate malignant lesions from non-malignant causes of restricted diffusion, a 'positive apparent diffusion (ADC) map' was determined as a decreased signal on the ADC coefficient with ADC reduction (15).

Upper gastrointestinal tract endoscopy, EUS and colonoscopy. The endoscopic devices used in the upper tract were the GIF-N260H, GIF-XP260NS, GIF-PG260, GIF-XQ260 and GIF-Q260 (Olympus, Tokyo, Japan). EUS was performed using GF-UCT260 (Olympus). The devices used for colonoscopy were the CF-Q260 and PCF-Q260AI (Olympus).

Statistical analysis. One-way analysis of variance or the Chi-squared test were applied using JMP 0.0.2 software (SAS Institute, Cary, NC, USA). Values are expressed as the mean \pm standard deviation.

\section{Results}

Patient characteristics. The patient details and diagnoses are summarized in Table II. Gastrointestinal tract cancers were 
Table II. List of patients diagnosed with cancer.

\begin{tabular}{|c|c|c|c|c|c|c|c|c|}
\hline $\begin{array}{l}\text { Patient } \\
\text { number }\end{array}$ & Diagnosis & T stage $^{\mathrm{a}}$ & $\begin{array}{l}\text { Depth of } \\
\text { invasion }\end{array}$ & $\begin{array}{l}\text { Diameter } \\
\quad(\mathrm{cm})\end{array}$ & $\mathrm{T} 1 \mathrm{~W}$ & $\mathrm{~T} 2 \mathrm{~W}$ & DWI & DWIBS/T2 \\
\hline 1 & Esophageal cancer & 3 & NA & NA & $(-)$ & $(-)$ & $(+)$ & $(+)$ \\
\hline 2 & Gastric cancer & $1 \mathrm{a}$ & M & 1.5 & $(-)$ & $(-)$ & $(-)$ & $(-)$ \\
\hline 3 & Gastric cancer & $1 \mathrm{a}$ & M & 1.3 & $(-)$ & $(-)$ & $(-)$ & $(-)$ \\
\hline 4 & Gastric cancer & $1 \mathrm{a}$ & M & 1.8 & $(-)$ & $(-)$ & $(-)$ & $(-)$ \\
\hline 5 & Gastric cancer & 2 & MP & 4 & $(-)$ & $(-)$ & $(+)$ & $(+)$ \\
\hline 6 & Gastric cancer & 3 & SS & 1.3 & $(-)$ & $(-)$ & $(+)$ & $(+)$ \\
\hline 7 & Gastric cancer & 4 & NA & NA & $(-)$ & $(-)$ & $(+)$ & $(+)$ \\
\hline 8 & Gastric cancer & 3 & NA & NA & $(-)$ & $(-)$ & $(+)$ & $(+)$ \\
\hline 9 & Gastric cancer & 3 & NA & NA & $(-)$ & $(+)$ & $(+)$ & $(+)$ \\
\hline 10 & Gastric cancer & 3 & NA & NA & $(-)$ & $(-)$ & $(+)$ & $(+)$ \\
\hline 11 & Gastric cancer & 3 & NA & NA & $(-)$ & $(-)$ & $(+)$ & $(+)$ \\
\hline 12 & Gastric cancer & $4 \mathrm{~b}$ & SS & 5 & $(-)$ & $(-)$ & $(+)$ & $(+)$ \\
\hline 13 & Gastric cancer & $3 a$ & MP & 6 & $(-)$ & $(-)$ & $(+)$ & $(+)$ \\
\hline 14 & Duodenal cancer & is & NA & NA & $(-)$ & $(-)$ & $(-)$ & $(-)$ \\
\hline 15 & Duodenal cancer & 2 & NA & NA & $(-)$ & $(-)$ & $(+)$ & $(+)$ \\
\hline 16 & Colon cancer ${ }^{\mathrm{b}}$ & is & $\mathrm{M}$ & 1.5 & $(+)$ & $(+)$ & $(+)$ & $(+)$ \\
\hline
\end{tabular}

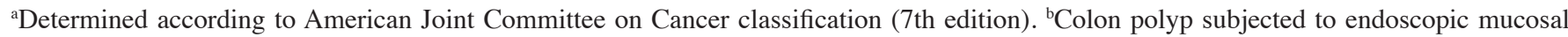
resection. T1W, T1-weighted image; T2W, T2-weighted image; DWI, diffusion-weighted image; DWIBS/T2, diffusion-weighted whole-body imaging with background body signal suppression/T2-weighted image fusion; NA, not analyzed; M, mucosa; MP, muscularis propria; SS, subserosa; (+), positive result; (-), negative result; is, in situ.

initially diagnosed by endoscopy (Fig. 1A). T staging was performed based on CE-CT and other diagnostic imaging techniques (Fig. 1B). Colon cancer was detected by T1WI as it was a polyp protruding into the lumen. T2WI was positive in one patient with gastric cancer and one patient with colon cancer. A thickened wall was identified by T1WI and T2WI in some cases; however, it was difficult to diagnose the other lesions as cancerous, as their intensities were identical to that of the surrounding tissues. A total of 12 patients were detected with DWI or DWIBS/T2. DWI and DWIBS/T2 were more sensitive compared with T2WI alone (Fig. 1C and D). DWI and DWIBS/T2 exhibited a significant contrast and had the same sensitivity. With DWIBS/T2 it was easier to analyze the strong positive signal in an anatomical context (Fig. 1E). Three patients with gastric cancer who were negative on DWIBS/T2, were found to be stage T1a and 1 patient with duodenal cancer who was negative on DWIBS/T2, was staged as Tis. The mechanism underlying the negative results on DWIBS/T2 is intriguing. The shape of the positive signal on DWIBS/T2 was consistent with that of the surgical specimen (Fig. 1F).

Association of detectability with $T$ stage. Subsequently, we focused on $\mathrm{T}$ staging and the association between tumor detectability with DWIBS/T2 and T stage was analyzed (Table III). All cancers staged $>\mathrm{T} 2$ were detectable by DWIBS/T2 and all cancers staged $<\mathrm{T} 1$ were not, clearly indicating that advanced cancer stage is significantly associated with its detectability with DWIBS/T2 ( $\mathrm{P}<0.0001)$.
Association of detectability with depth of invasion. The association between the tumor diameter and detectability was next analyzed. Diameters were plotted against detection with DWIBS/T2 (Fig. 2). The mean diameter of tumors not detected by DWIBS/T2 was $1.53 \pm 0.25 \mathrm{~cm}$, while that of detected tumors was $3.63 \pm 1.88 \mathrm{~cm}$; however, the difference was not statistically significant $(\mathrm{P}=0.1053)$.

Furthermore, the association of depth of invasion of the tumors with their detectability by DWIBS/T2 was assessed (Table IV). All 5 cancers that had invaded beyond the muscularis propria were detected by DWIBS/T2, whereas 3 cases that had not invaded the mucosa were not detected. The depth of invasion was significantly associated with detectability by DWIBS/T2 $(\mathrm{P}=0.0476)$. Colon cancer was positive on DWIBS/T2, although was confined in the mucosa; the cancer was originally a colon polyp with a diameter of $1.5 \mathrm{~cm}$.

\section{Discussion}

Until recently, DWI or DWIBS with a 1.5-Tesla scanner was considered to be unsuitable for imaging of abdominal organs due to respiratory movement (16-18). However, the protocol of acquiring images has improved with the use of a respiratory trigger (6). In the present study, all gastric and duodenal cancers staged $>$ T2 were detectable by DWI and DWIBS/T2 (19). DWI and DWIBS/T2 exhibited a strong signal and contrast against the surrounding tissues. For this reason, DWI and DWIBS/T2 had better sensitivity when compared with T2WI alone. Unlike endoscopy, DWIBS/T2 may be useful for evaluating the extent 

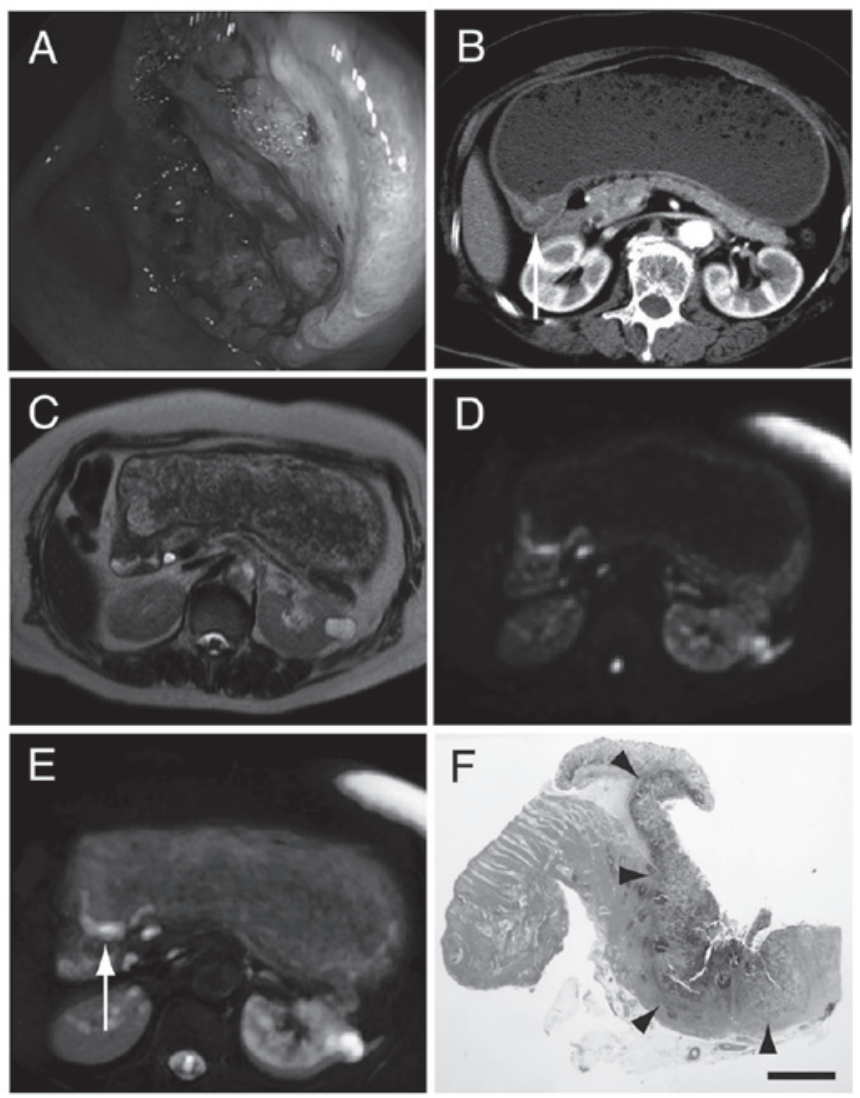

Figure 1. Representative images from a patient with gastric cancer. A 74-year-old woman with significant vomiting was subjected to (A) upper gastrointestinal endoscopy, revealing an irregularly shaped ulcer obstructing the pylorus. The patient's stomach was filled with fluid and (B) the wall near the pylorus was found to be thickened on contrast-enhanced computed tomography. (C) T2-weighted imaging and (D) diffusion-weighted whole-body imaging with background body signal suppression (DWIBS) were fused to create (E) a DWIBS/T2 fusion image showing a strong signal in the thickened part of the gastric wall (arrow). (F) Gastric cancer was diagnosed in the hematoxylin and eosin-stained sections from the thickened gastric wall (arrowheads). Scale bar, $5 \mathrm{~mm}$.

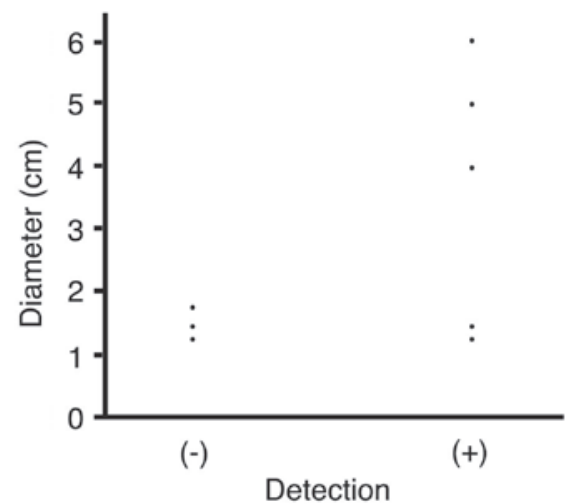

Figure 2. Plots showing the diameters of gastric or colorectal cancer in association with detectability by diffusion-weighted whole-body imaging background body signal suppression/T2-weighted fusion image (DWIBS/T2). (+), detected by DWIBS/T2; (-), not detected by DWIBS/T2.

and depth of invasion of gastric cancer (20-22). By contrast, all gastric and duodenal cancers exhibiting invasion of $<\mathrm{T} 1$ were not detected by DWIBS/T2, indicating that $\mathrm{T}$ stage affected tumor detectability by DWIBS/T2. In particular, Borrmann 4
Table III. Correlation between tumor detectability by DWIBS/T2 and T stage.

\begin{tabular}{lrrr}
\hline & \multicolumn{2}{c}{ T stage $^{\mathrm{a}}$} & \\
\cline { 2 - 3 } Detection & $>\mathrm{T} 2$ & $<\mathrm{T} 1$ & Total \\
\hline$(+)$ & 12 & 0 & 12 \\
$(-)$ & 0 & 4 & 4 \\
Total & 12 & 4 & 16
\end{tabular}

a Determined according to the American Joint Committee on Cancer classification (7th edition); (+), positive results; $(-)$, negative results. $\mathrm{P}<0.0001$ (Chi-squared test). DWIBS/T2, diffusion-weighted wholebody imaging background body signal suppression/T2-weighted fusion image.

Table IV.Association between tumor detectability by DWIBS/T2 and depth of invasion.

\begin{tabular}{llll}
\hline & \multicolumn{2}{c}{ Depth of invasion } & \\
\cline { 2 - 3 } Detection & $>$ MP & M & Total \\
\hline$(+)$ & 5 & $1^{\text {a }}$ & 5 \\
$(-)$ & 0 & 3 & 4 \\
Total & 5 & 4 & 9
\end{tabular}

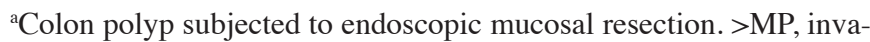
sion beyond muscularis propria; $\mathrm{M}$, invasion confined to mucosa; (+), positive results; (-), negative results; $\mathrm{P}=0.0476$ (Chi-squared test). DWIBS/T2, diffusion-weighted whole-body imaging background body signal suppression/T2-weighted fusion image.

gastric cancer exhibits a thickened wall,referred to as 'sandwich sign' (23). Our findings suggested that DWIBS/T2 may add diagnostic information to the process of T-staging (20-22,24).

$\mathrm{T}$ staging is performed based on the depth of invasion regarding gastrointestinal tract cancers. The present study revealed that cancers confined within the mucosa were not detected by DWIBS/T2. One exception was a case of colon cancer; the patient presented with a colon polyp and underwent endoscopic mucosal resection. The polyp was $1.5 \mathrm{~cm}$ in diameter and protruded into the lumen. It was hypothesized that the polyp was of sufficient size to be detectable by DWIBS/T2, even though the cancer had only invaded the mucosa. Of note, all other cancers that were not detectable by DWIBS/T2 were flat. Cancers confined within the mucosa may be positive on DWIBS/T2 upon reaching a certain volume. High-spatial resolution MRI is able to detect gastric cancer within the mucosa (22). However, this technique is currently not applied.

One limitation of the present study was the small number of patients. Further studies including more colon and duodenal cancer patients are required to confirm our findings. Another limitation was that tumor invasion of the muscularis propria (PM), subserosa (SS) and serosal exposure (SE) was not analyzed. In future studies, the possibility to differentiate 
between PM, SS and SE invasion with DWIBS/T2 compared with endoscopic ultrasound should be addressed (25).

In conclusion, DWIBS/T2 was able to identify gastrointestinal cancers staged as $>\mathrm{T} 2$ or invading beyond the muscularis propria.

\section{References}

1. Allum WH, Blazeby JM, Griffin SM, Cunningham D, Jankowski JA and Wong R; Association of Upper Gastrointestinal Surgeons of Great Britain and Ireland, the British Society of Gastroenterology and the British Association of Surgical Oncology: Guidelines for the management of oesophageal and gastric cancer. Gut 60: 1449-1472, 2011.

2. Labianca R, Nordlinger B, Beretta GD, Mosconi S, Mandalà M, Cervantes A and Arnold D; ESMO Guidelines Working Group: Early colon cancer: ESMO clinical practice guidelines for diagnosis, treatment and follow-up. Ann Oncol 24 (Suppl 6): vi64-vi72, 2013.

3. Didden P, Spaander MC, Wijnhoven BP, Kuipers EJ and Bruno MJ: Improving the quality of pretreatment staging in patients with esophageal carcinoma-a fast track study. Acta Oncol 51: 362-367, 2012

4. Umeoka S, Koyama T, Togashi K, Saga T, Watanabe G, Shimada Y and Imamura M: Esophageal cancer: Evaluation with triple-phase dynamic CT-initial experience. Radiology 239: 777-783, 2006.

5. Choi JI, Joo I and Lee JM: State-of-the-art preoperative staging of gastric cancer by MDCT and magnetic resonance imaging. World J Gastroenterol 20: 4546-4557, 2014

6. Takahara T, Imai Y, Yamashita T, Yasuda S, Nasu S and Van Cauteren M: Diffusion weighted whole body imaging with background body signal suppression (DWIBS): Technical improvement using free breathing, STIR and high resolution 3D display. Radiat Med 22: 275-282, 2004.

7. Sehy JV, Ackerman JJ and Neil JJ: Apparent diffusion of water, ions, and small molecules in the Xenopus oocyte is consistent with Brownian displacement. Magn Reson Med 48: 42-51, 2002.

8. Koike N, Cho A, Nasu K, Seto K, Nagaya S, Ohshima Y and Ohkohchi N: Role of diffusion-weighted magnetic resonance imaging in the differential diagnosis of focal hepatic lesions. World J Gastroenterol 15: 5805-5812, 2009.

9. Kwee TC, Takahara T, Ochiai R, Nievelstein RA and Luijten PR: Diffusion-weighted whole-body imaging with background body signal suppression (DWIBS): Features and potential applications in oncology. Eur Radiol 18: 1937-1952, 2008.

10. Ohno Y, Koyama H, Onishi Y, Takenaka D, Nogami M, Yoshikawa T, Matsumoto S, Kotani Y and Sugimura K: Non-small cell lung cancer: Whole-body MR examination for M-stage assessment-utility for whole-body diffusion-weighted imaging compared with integrated FDG PET/CT. Radiology 248: 643-654, 2008.

11. Fischer MA, Nanz D, Hany T, Reiner CS, Stolzmann P, Donati OF, Breitenstein S, Schneider P, Weishaupt D, von Schulthess GK and Scheffel H: Diagnostic accuracy of whole-body MRI/DWI image fusion for detection of malignant tumours: A comparison with PET/CT. Eur Radiol 21: 246-255, 2011.
12. Sommer G, Wiese M, Winter L, Lenz C, Klarhöfer M, Forrer F, Lardinois D and Bremerich J: Preoperative staging of non-small-cell lung cancer: Comparison of whole-body diffusion-weighted magnetic resonance imaging and $18 \mathrm{~F}$-fluorodeoxyglucose-positron emission tomography/computed tomography. Eur Radiol 22: 2859-2867, 2012.

13. Nechifor-Boilă IA, Bancu S, Buruian M, Charlot M, Decaussin-Petrucci M, Krauth JS, Nechifor-Boilă AC and Borda A: Diffusion weighted imaging with background body signal suppression/T2 image fusion in magnetic resonance mammography for breast cancer diagnosis. Chirurgia (Bucur) 108: 199-205, 2013.

14. Washington K: 7th edition of the AJCC cancer staging manual: Stomach. Ann Surg Oncol 17: 3077-3079, 2010.

15. Wang Y, Miller FH, Chen ZE, Merrick L, Mortele KJ, Hoff FL, Hammond NA, Yaghmai V and Nikolaidis P: Diffusion-weighted MR imaging of solid and cystic lesions of the pancreas. Radiographics 31: E47-E64, 2011.

16. Mürtz P, Krautmacher C, Träber F, Gieseke J, Schild HH and Willinek WA: Diffusion-weighted whole-body MR imaging with background body signal suppression: A feasibility study at 3.0 Tesla. Eur Radiol 17: 3031-3037, 2007.

17. Caivano R, Rabasco P, Lotumolo A, D' Antuono F, Zandolino A, Villonio A, Macarini L, Guglielmi G, Salvatore M and Cammarota A: Gastric cancer: The role of diffusion weighted imaging in the preoperative staging. Cancer Invest 32: 184-190, 2014.

18. De Cobelli F, Giganti F, Orsenigo E, Cellina M, Esposito A, Agostini G, Albarello L, Mazza E, Ambrosi A, Socci C, et al: Apparent diffusion coefficient modifications in assessing gastro-oesophageal cancer response to neoadjuvant treatment: Comparison with tumour regression grade at histology. Eur Radiol 23: 2165-2174, 2013

19. Huo X, Yuan K, Shen Y, Li M, Wang Q, Xing L and Shi G: Clinical value of magnetic resonance imaging in preoperative $\mathrm{T}$ staging of gastric cancer and postoperative pathological diagnosis. Oncol Lett 8: 275-280, 2014.

20. Mocellin S, Marchet A and Nitti D: EUS for the staging of gastric cancer: A meta-analysis. Gastrointest Endosc 73: 1122-1134, 2011.

21. Bohle W, Scheidig A and Zoller WG: Endosonographic tumor staging for treatment decision in resectable gastric cancer. J Gastrointestin Liver Dis 20: 135-139, 2011.

22. Yamada I, Saito N, Takeshita K, Yoshino N, Tetsumura A, Kumagai J and Shibuya H: Early gastric carcinoma: Evaluation with high-spatial-resolution MR imaging in vitro. Radiology 220: $115-121,2001$.

23. Zhang XP, Tang L, Sun YS, Li ZY, Ji JF, Li XT, Liu YQ and Wu Q: Sandwich sign of Borrmann type 4 gastric cancer on diffusion-weighted magnetic resonance imaging. Eur J Radiol 81: 2481-2486, 2012

24. Liu S, He J, Guan W, Li Q, Yu H, Zhou Z, Bao S and Zhou Z: Added value of diffusion-weighted MR imaging to T2-weighted and dynamic contrast-enhanced MR imaging in $\mathrm{T}$ staging of gastric cancer. Clin Imaging 38: 122-128, 2014.

25. Lei C, Huang L, Wang Y, Huang Y and Huang Y: Comparison of MRI and endoscope ultrasound detection in preoperative T/N staging of gastric cancer. Mol Clin Oncol 1: 699-702, 2013. 